\title{
Glans ischemia following inflatable penile prosthesis surgery
}

\author{
Kevin J. Hebert, Tobias S. Kohler \\ Department of Urology, Mayo Clinic, Rochester, MN, USA \\ Correspondence to: Tobias S. Kohler. Department of Urology, Mayo Clinic, Rochester, MN 55905, USA. Email: Kohler.Tobias@mayo.edu.
}

\begin{abstract}
We present a case report of acute glans ischemia in a patient with significant vascular comorbidities following insertion of an inflatable penile prosthesis for erectile dysfunction. In this report, we pictorially display the physical exam findings that led to prompt diagnosis and discuss the importance of early device explantation (within 24 hours) to prevent glandular tissue loss.
\end{abstract}

Keywords: Anticoagulation; erectile dysfunction; inflatable penile prosthesis (IPP); glans ischemia; glans necrosis; post-IPP ischemia

Submitted Sep 21, 2019. Accepted for publication Dec 09, 2019.

doi: 10.21037/tau.2020.01.04

View this article at: http://dx.doi.org/10.21037/tau.2020.01.04

\section{Introduction}

Glans ischemia is an uncommon post-operative complication following inflatable penile prosthesis (IPP) surgery. To date, less than twenty-one cases have been reported in the literature (1-4). Its rarity, combined with likely underreporting, has led to a paradigm of non-guideline based management. We present, to our knowledge, the first complete pictorially documented case of post-IPP glans ischemia adapted from Hebert and Kohler published in Fournal of Sexual Medicine (5), with the goal to continue open discussion of this rare complication in hopes to provide clarity regarding appropriate management for surgeons.

\section{Case presentation}

A 27-year-old male presented to our clinic with medically refractory erectile dysfunction precluding penetrative intercourse. His medical history was extensive, including poorly controlled insulin-dependent diabetes (pre-operative HgA1c of 9.0, on insulin pump), diabetic retinopathy, nonST segment myocardial infarction at the age of 25 status post drug-eluting stent placement to three arteries (right circumflex artery, proximal right coronary artery, middle right coronary artery), peripheral artery disease status post superficial femoral artery (SFA) stenting with subsequent complete occlusion of the right SFA stent resulting in acute limb ischemia and dry gangrene of the third digit, status post right common iliac artery to profunda femoris bypass graft and common femoral artery to below-knee popliteal artery Dacron bypass graft, on triple anti-thrombotic therapy including aspirin $81 \mathrm{mg}$, warfarin, and clopidogrel. Phosphodiesterase inhibitors were contraindicated due to concurrent use of isosorbide mononitrate for angina. He was initiated on papaverine/phentolamine injection therapy for 5 months which failed and returned to our clinic to discuss the role of penile prosthesis surgery with goals to achieve a successful pregnancy with his partner. Our patient had no history of penile curvature or prior abdominal surgery. His social history was pertinent for a 24 pack-year smoking history (2 packs per day for 12 years). Physical exam was unremarkable with a circumcised phallus and bilateral descended testicles. After a detailed discussion regarding the increased risk of surgery secondary to his extensive cardiovascular history and poorly controlled diabetes, he elected to pursue IPP surgery. Preoperative evaluation by primary care and cardiology included review of recent stress echocardiogram and stress EKG testing which showed no evidence of ischemia. Surgical clearance was granted and after a consultation with thrombophilia clinic, he was continued on aspirin/clopidogrel and bridged from warfarin with enoxaparin.

Uneventful IPP surgery was performed via penoscrotal approach with placement of bilateral Coloplast ${ }^{\circledR}$ (Minneapolis, MN) $20 \mathrm{~cm}$ cylinders. A 75-cc reservoir was 

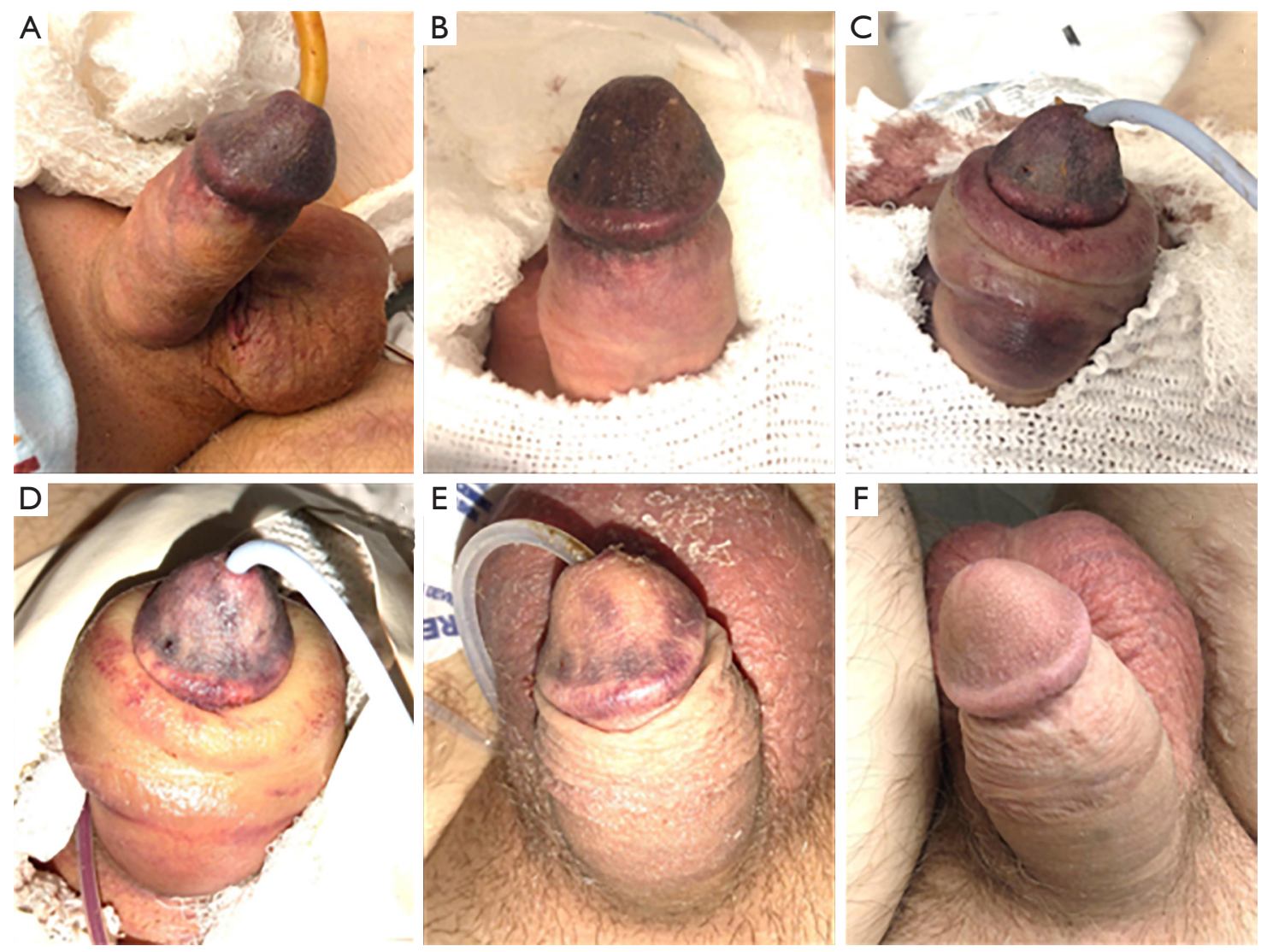

Figure 1 Post-operative progression/improvement of glans ischemia. Adapted from Hebert K, Kohler TS. Acute Post-Inflatable Penile Prosthesis Glans Ischemia: Review of Incidence, Pathophysiology, and Management Recommendations. J Sex Med 2019;16:1-4 (5). (A) Appearance at 8 hours post-operatively; (B) appearance on POD 1 (17 h post op); (C) appearance on POD 1 s/p device removal; (D) appearance on POD $3 \mathrm{~s} / \mathrm{p}$ device removal; (E) appearance on POD $9 \mathrm{~s} / \mathrm{p}$ device removal; (F) appearance on POD $21 \mathrm{~s} / \mathrm{p}$ device removal. POD, Post operative day.

placed in the left retropubic space. Our standard practice is to leave a 10 Fr Jackson Pratt drain tracking along the reservoir tubing into the dependent portion of the scrotum. The device was left $60-80 \%$ inflated and a 4 -inch kerlix was used for a mummy wrap (6) to compress the scrotum. The scrotum was supported with a jock strap. A 16 French Foley catheter with $10 \mathrm{cc}$ in the balloon, placed at the start of the case, was left in place at case completion. Total intraoperative time was $40 \mathrm{~min}$.

Due to the risk of a post-operative cardiovascular event, our patient was restarted on therapeutic enoxaparin $2 \mathrm{~h}$ postoperatively. We were contacted $8 \mathrm{~h}$ post-operatively with concern for a "dusky glans." On immediate evaluation, the glans penis appeared to have vascular compromise, but was without glans blistering (Figure 1A). The patient reported pain at the penile tip with intact sensation. The device was deflated, Foley catheter was removed, and the mummy wrap was taken down. At re-evaluation $1 \mathrm{~h}$ later, our patient reported improvement in pain and moderate improvement in coloration. We elected to perform serial exams overnight with plan for urgent device explantation on the morning of post-operative day one, if substantial improvement in coloration was not appreciated. At 5 am on postoperative day one (17 h post op), re-assessment revealed no considerable change in glans viability (Figure 1B). After discussion of risks of continued conservative management (glans tissue loss, device erosion, infection, and eventual need for explantation), we proceeded with emergent device explant via penoscrotal approach. At this point, patient was fully anticoagulated with aspirin $81 \mathrm{mg}$, clopidogrel $75 \mathrm{mg}$, and therapeutic enoxaparin as previously described. Intraoperatively, the bladder was drained with a 12 French Foley 


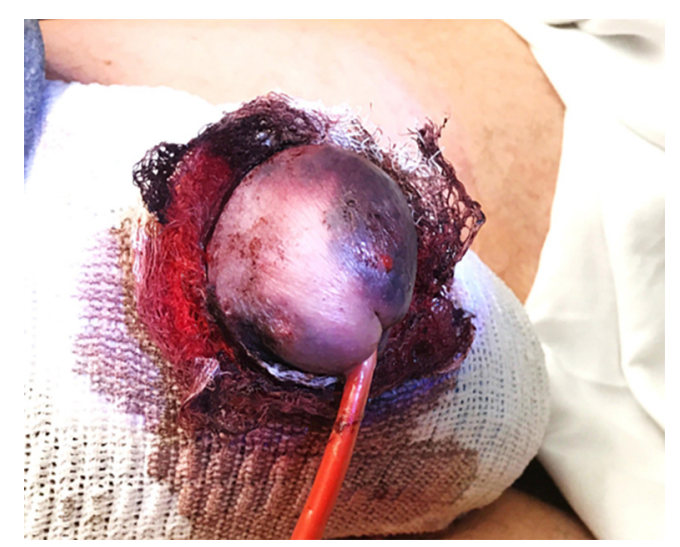

Figure 2 Post-operative glans bruising surrounding Keith needle puncture site (arrow). Notice the incomplete glans discoloration seen with glans bruising versus complete glans discoloration as depicted in Figure $1 A$ seen with glans ischemia.

catheter. The device was removed in its entirety. No single site of bleeding was encountered, however diffuse tissue oozing was identified. We left a 10 French Jackson Pratt drain in the left external inguinal ring. A jock strap was left in place, however, we elected to not perform a penile mummy wrap. Post-operatively, our patient displayed elevated serosanguinous scrotal drain output of $\sim 100 \mathrm{cc}$ per hour, totaling $\sim 900 \mathrm{cc}$ over 10 hours. Serial hemoglobins down trended to 7.4, which required 1 unit of packed red blood cells (pRBCs). Vascular medicine was consulted for anticoagulation management recommendations in the setting of elevated drain output and risk of Dacron graft occlusion and recommended discontinuation of clopidogrel and Lovenox. On post-operative day 1 status post explant, the glans displayed continued ischemic appearance (Figure 1C). Significant penile shaft and scrotal swelling were also noted. Our patient's hemoglobin continued to downtrend despite minimal drain output which required transfusion of an additional two units of pRBCs. On post-operative day 2, we applied scrotal fluffs and foam tape to the perineum to increase scrotal pressure. Perioperative gentamicin and vancomycin were continued during this period. A baseline ultrasound of the lower extremity graft was performed to verify patency. No evidence of occlusion was present. The glans showed slow, but gradual improvement in viability (Figure 1D). On post-operative day 4, the patient reported new right calf pain, however lower extremity pulses were unchanged from the preoperative exam and a lower extremity ultrasound revealed no evidence of acute deep vein thrombosis. Prophylactic enoxaparin was restarted in the setting of stable hemoglobins and minimal drain output. The patient was discharged on post-operative day 5 following stable serial CBCs/vital signs and unchanged physical exam after being advanced to therapeutic enoxaparin. Due to a failed voiding trial prior to discharge, a 12 French Foley catheter was replaced. We elected to transition the patient to one week of trimethoprim/ sulfamethoxazole. At discharge, bruising remained primarily at the corona with improvement in the mid and distal glans. He was followed closely in the outpatient setting by thrombophilia for anticoagulation management. The patient returned to clinic for a wound check on post-operative day 9. This showed near complete resolution of discoloration (Figure 1E), and the patient underwent successful urinary catheter removal. He was again seen on post-operative day 21 in clinic for a wound check with complete glans viability noted (Figure $1 F$ ). A $1 \mathrm{~cm}$ area of skin separation was noted at our penoscrotal incision and was likely related to skin dehiscence secondary to increased scrotal pressure due to swelling. This was allowed to heal via secondary intention.

\section{Discussion}

Glans ischemia involves the entire glans with a uniform distinct color change between the corona and penile shaft. Additional physical exam findings include decreased glans sensation, increased pain, and skin blistering. Not to be confused with glans ischemia, significant bruising is common and can be present post-operatively due to intraoperative puncture of the glans with the Keith needle during distal seating of the device or from distal dissection (Figure 2).

However bruising of the glans from keith needle puncture can be delineated from glans ischemia by the distribution of glans discoloration. Glans ischemia produces discoloration of the entire glans whereas keith needle brusing produces incomplete discoloration of the glans.

If concern for glans ischemia is present, clinicians should counsel patients towards emergent explant of the device to reduce risk of glandular tissue loss. Sagacious words of wisdom include, "When in doubt, take it out." Patients who undergo removal are often able to undergo reattempt of device placement at a later date. Furthermore, Wilson et al. (1) showed that no patients experienced tissue loss if the device was explanted within $24 \mathrm{~h}$ of glans ischemia onset (1). To our knowledge, all reports of a wait and see approach to this problem have resulted in catastrophic penile tissue loss and eventual device removal. 
Risk factors for glans ischemia are intuitive, including classic factors that could compromise the urethral blood supply to the glans such as diabetes, smoking, and history of vascular disease. Although his diabetes was poorly controlled, our patient met with endocrinology who felt he was optimized with an insulin pump. Maneuvers that increase extrinsic compression (compressive penile dressings or max inflated cylinders) or compromise urethral blood flow internally (urethral dissection/mobilization and use of a large bore catheter) can exacerbate the problem. Our patient met with our thrombophilia colleagues prior to surgery and continued anticoagulation after a discussion of risks and benefits of withholding peri-procedural anticoagulation to minimize the risk of Dacron graft occlusion. Increased morbidity related to his transfusion requirement only presented after removal of the device (we routinely perform IPP cases on anticoagulation without ever having had to transfuse previously). Intra-operatively, consideration of narrower inflatable cylinders could have reduced the radial pressure to the penis (6). Decreasing catheter size, performing CIC, and/or leaving the device deflated may have been helpful.

Our nursing team displayed excellent clinical acuity which directly impacted the outcome of our patient's care. Continued partnership and education of nurses and physicians with post-operative IPP patients is integral to ensure timely recognition and management of this complication which must be addressed within 24 hours of onset.

Acute glans ischemia remains a difficult post-operative complication to manage due to the need for immediate, invasive intervention as well as a paucity guideline-based management. This case presented multiple complexities due to the patient's numerous comorbidities; however, it highlights the importance of early consideration, recognition, and immediate removal of the device if glans ischemia is suspected.

\section{Acknowledgments}

Funding: None.

\section{Footnote}

Conflicts of Interest: TSK serves as an unpaid Associate Editor-in-Chief of Translational Andrology and Urology from Jan 2020 to Dec 2021. The other authors have no conflicts of interest to declare.

Ethical Statement: The authors are accountable for all aspects of the work in ensuring that questions related to the accuracy or integrity of any part of the work are appropriately investigated and resolved. Written informed consent was obtained from the patient for publication of this manuscript and any accompanying images.

Open Access Statement: This is an Open Access article distributed in accordance with the Creative Commons Attribution-NonCommercial-NoDerivs 4.0 International License (CC BY-NC-ND 4.0), which permits the noncommercial replication and distribution of the article with the strict proviso that no changes or edits are made and the original work is properly cited (including links to both the formal publication through the relevant DOI and the license). See: https://creativecommons.org/licenses/by-ncnd/4.0/.

\section{References}

1. Wilson SK, Mora-Estaves C, Egydio P, et al. Glans Necrosis Following Penile Prosthesis Implantation: Prevention and Treatment Suggestions. Urology 2017;107:144-8.

2. Shelling RH, Maxted WC. Major complications of silicone penile prosthesis: predisposing clinical situations. Urology 1980;15:131-3.

3. Bejany DE, Perito PE, Lustgarten M, Rhamy RK. Gangrene of the penis after implantation of penile prosthesis: case reports, treatment recommendations and review of the literature. J Urol 1993;150:190-1; discussion 191-2.

4. García Gómez B, Romero Otero J, Díez Sicilia L, et al. Ischemic Gangrene of the Glans following Penile Prosthesis Implantation. Case Rep Urol 2013;2013:323574.

5. Hebert K, Kohler TS. Acute Post-Inflatable Penile Prosthesis Glans Ischemia: Review of Incidence, Pathophysiology, and Management Recommendations. J Sex Med 2019;16:1-4.

6. Henry GD. The Henry mummy wrap and the Henry finger sweep surgical techniques. J Sex Med 2009;6:619-22.

Cite this article as: Hebert KJ, Kohler TS. Glans ischemia following inflatable penile prosthesis surgery. Transl Androl Urol 2020;9(2):824-827. doi: 10.21037/tau.2020.01.04 